\title{
APLICAÇÃO DE VEÍCULOS AÉREOS NÃO TRIPULADOS NA OBTENÇÃO DE PARÂMETROS PARA ANÁLISE DA PAISAGEM: UM ENSAIO DE PESQUISA EM CAMPO VERDE - MT
}

\author{
Cleberson Ribeiro de Jesuz ${ }^{(a)}$, Francisco de Assis Gonçalves Junior ${ }^{\left({ }^{(b)}\right.}$,Manuel Eduardo Ferreira $^{(\mathrm{c})}$, \\ Roberto Nunes Vianconi Souto ${ }^{(\mathrm{d})}$ \\ (a) Docente no Departamento de Geografia/Instituto de Geografia, História e Documentação, Universidade Federal de \\ Mato Grosso, E-mail:cleberufmt@hotmail.com \\ (b) Docente no Departamento de Geografia/Instituto de Geografia, História e Documentação, Universidade Federal de \\ Mato Grosso, E-mail: fgjufmt@gmail.com. \\ (c) Docente do Instituto de Estudos Sócio-Ambientais/Programa de Pós-Graduação em Geografia, Universidade \\ Federal de Goiás, Campus Goiânia, E-mail: mferreira.geo@gmail.com. \\ (d) Docente no Departamento da Área de Construção Civil, Instituto Federal de Educação, Ciência e Tecnologia de \\ Mato Grosso, Campus Cuiabá, E-mail: vianconisouto@ hotmail.com
}

\section{Eixo: GEOTECNOLOGIAS E MODELAGEM ESPACIAL EM GEOGRAFIA FÍSICA}

\begin{abstract}
Resumo
O uso de novas técnicas e tecnologias para análise de paisagens nativas e antrópicas vêm ganhando importância nos últimos anos, especialmente o emprego de Veículos Aéreos Não Tripulados (VANT), equipados com sensores imageadores. Neste ensaio, um VANT (modelo Swinglet CAM) foi utilizado para sobrevoar duas nascentes de primeira ordem, situadas em duas propriedades rurais em Campo Verde - MT, com uso e ocupação da terra distintas (agricultura e pastagem), formadoras de uma linha interfluvial subsidiadas pela ação de um colo topográfico. A metodologia também envolveu uma câmera digital RGB (12 MP) a bordo do VANT, totalizando 10 planos de voo em agosto de 2016. A alta resolução espacial do mosaico ortorretificado (Modelo Digital de Superfície $-10 \mathrm{~cm}$ ), associado a técnicas de geoprocessamemto e GNSS (pontos de apoio em campo), foi possível mapear algumas métricas da paisagem, tais como hipsometria, declividade e uso do solo, subsidiando análises secundárias na área de estudo.
\end{abstract}

Palavras-chave: VANT; Geotecnologias; Análise da Paisagem; Modelo Digital de Superfície.

\section{Introdução}

As sociedades humanas apresentam maneiras específicas de organização e apropriação dos recursos naturais, invariavelmente impactantes, em menor ou maior grau. Quando os sistemas produtivos são inadequados, estes podem gerar impactos e consequências socioambientais ainda maiores e intensos, variando de acordo com a apropriação e o manejo dos recursos ao longo do tempo.

Neste contexto, as áreas rurais apresentam-se como as mais vulneráveis aos sistemas produtivos inadequados, pois sua dinâmica de uso, voltada às demandas mercadológicas por quantidade e qualidade, choca-se com o tempo de resiliência natural do sistema, desencadeando problemas ambientais já bem descritos na literatura científica. 
A partir desta premissa, e considerando a possibilidade de construção de um diagnóstico amplo e preciso voltado à prevenção de impactos ambientais sobre as áreas rurais, novas pesquisas vêm se debruçando sobre a paisagem enquanto categoria de análise, uma vez que sua perspectiva sistêmica e integrada possibilita o aprofundamento de estudos voltados ao planejamento ambiental.

Para Monteiro (2001), a questão metodológica fundamental para o discernimento das paisagens é a das escalas espaço-temporais, ou seja, a ação dos elementos ao longo do tempo, em diferentes escalas, com a interferência da ação humana, variando de escalas menores para maiores. Assim, a diversidade espaçotemporal na paisagem se baseia na inter-relação das diferentes formas de uso e ocupação com as características naturais da paisagem, constituindo um sistema integrado. Por sua vez, através do diagnóstico da paisagem, onde são reconhecidos os seus elementos principais, sua estrutura e seu funcionamento, podem-se criar mecanismos mais hábeis para subsidiar as ações voltadas ao planejamento ambiental.

Nesse sentido, consideram-se as possibilidades técnicas advindas do sensoriamento remoto na elaboração de diagnósticos mais precisos sobre as paisagens, especialmente no meio rural. O sensoriamento remoto, seja este orbital ou aéreo, através de ferramentas específicas do Geoprocessamento, tem possibilitado a integração de diversos elementos/atributos da paisagem, tais como solos, morfometria, dados climáticos, hidrografia, uso e ocupação da terra, etc. (WWF, 2009, 2011; SCHMUGGE, 2002; LATRUBESSE et al., 1998; JESUZ et al., 2013; BOEGH et al., 2004; SANO et al., 1993; REATTO et al., 2004).

Dentre estas técnicas de sensoriamento remoto, o uso de sensores imageadores a bordo de Veículos Aéreos Não Tripulados (VANT) tem se destacado particularmente na última década, se consolidando como uma nova forma de obtenção de parâmetros sub-orbitais de alta qualidade espacial, espectral e multisensorial (ALMEIDA, 2010; DIAS e ROSSA, 2015; COLOMINA e MOLINA, 2014). Sua versatilidade logística e de aplicações, aliada a custos relativamente baixos, vem estimulando o emprego dos VANTs nos estudos geoambientais em geral (OUÉDRAOGO et al., 2014; NIETHAMMER et al., 2012; NIKOLAKOPOULOS et al., 2015; FLENER et al., 2013; NÄSI et al., 2015; DANDOIS et al., 2015; LUCIEER et al., 2013).

Nessa pesquisa, considerando-se a paisagem como categoria de análise, bem como o VANT como uma ferramenta ideal para descreve-la em termos espaço-temporais, busca-se a realização de um ensaio sobre área rural pré-definida no município de Campo Verde - MT, no intuito de constituir um Modelo Digital de Superfície (MDS) com alta resolução espacial $(10 \mathrm{~cm})$, como subsídio para um diagnóstico mais preciso, principalmente no que se refere à dinâmica de fluxos na superfície (radiação e umidade) em vertentes com formas de uso e ocupação distintas (pastagem e agricultura). 


\section{Materiais e Métodos}

\subsection{Recorte espacial}

O referido ensaio foi conduzido sobre uma área interfluvial situada na região sudeste do Estado de Mato Grosso, entre duas cabeceiras de nascentes de primeira ordem do rio das Mortes, no município de Campo Verde. A área delimitada possui cerca de $7,86 \mathrm{~km}^{2}$, distribuídos em duas fazendas com uso diferenciados, sendo uma com pecuária extensiva (ao sul), e outra com agricultura mecanizada (soja/milho/algodão), (ao norte). A área está localizada entre as Latitudes Sul $-15^{\circ} 28^{\prime} 30^{\prime \prime}$ e $-15^{\circ} 31^{\prime} 44^{\prime \prime}$, e as Longitudes Oeste $-55^{\circ} 16^{\prime} 12^{\prime \prime}$ e $-55^{\circ} 13$ ' 33” (Fig. 1).

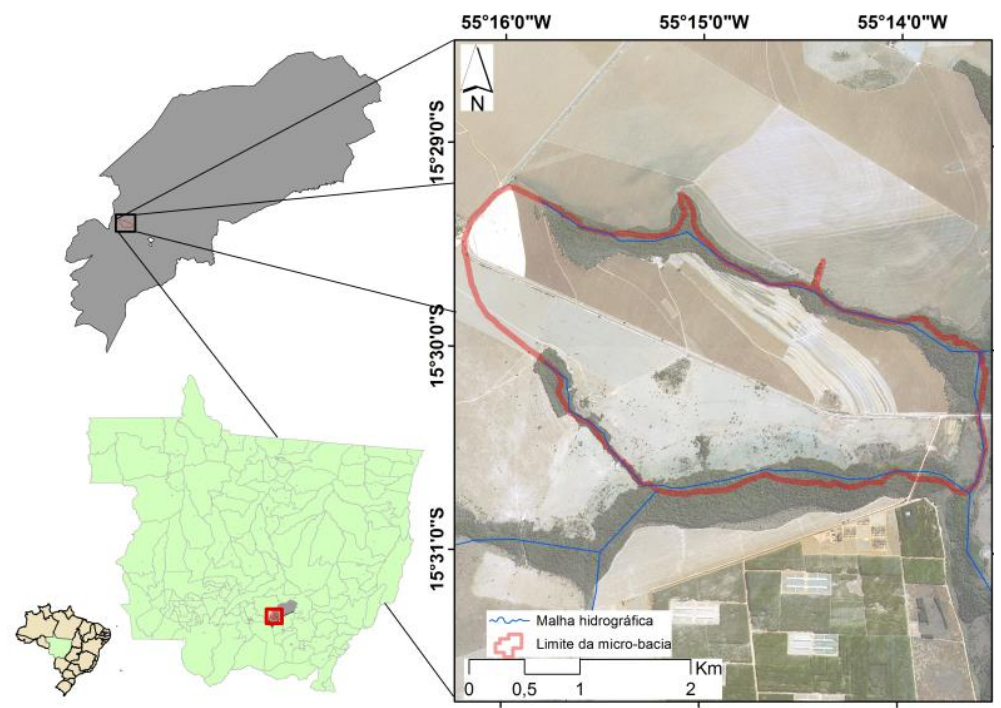

Figura 1. Localização da área de estudo, com destaque às drenagens de primeira ordem analisadas. Dados obtidos na pesquisa, Imagens SPOT TM-5 (Agost. 2007).

\subsection{Procedimentos Técnico-operacionais}

Os levantamentos aéreos deste ensaio foram realizados em duas etapas de campo: nos dias 03 (voos 1 a 5 ) e 04 de agosto (voos 5 a 10) de 2016, empregando-se um Veículo Aéreo Não Tripulado da Universidade Federal de Goiás/IESA/LAPIG. O modelo de VANT utilizado foi o Swinglet CAM, fabricado pela empresa suíça Sensefly (https://www.sensefly.com/home.html). O plano de voo foi realizado no programa Emotion (Electronic Monitoring Station), enquanto que o Modelo Digital de Superfície (MDS) e os ortomosaicos foram confeccionados no programa Pix4D Mapper (https://pix4d.com). Ao todo, foram realizados 10 sobrevoos na área, a fim de recobrir o objeto de estudo, conforme ilustrado na Figura 1. 


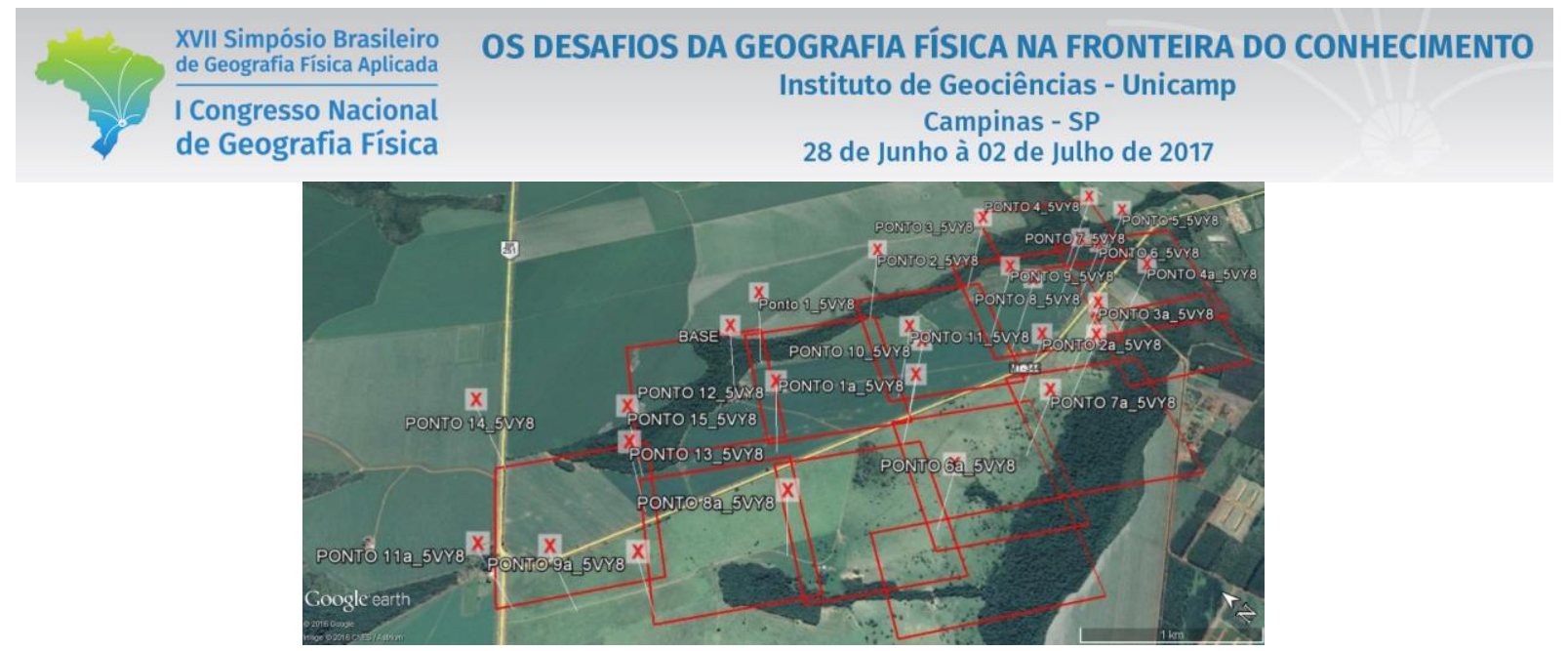

Figura 2. Distribuição espacial dos 10 planos de voos necessários ao recobrimento da área de estudo, com resolução espacial (ou GSD) de $10 \mathrm{~cm}$.

Sobre o Swinglet CAM, trata-se de um microvant (i.e., abaixo de $25 \mathrm{~kg}$ ) do tipo asa-fixa, com propulsor elétrico, projetado para voar somente em boas condições climáticas, suportando ventos com velocidade inferior a $25 \mathrm{~km} / \mathrm{h}$ (aproximadamente $7 \mathrm{~m} / \mathrm{s}$ ). O Swinglet e seus componentes estão representados na Figura 3. Nota-se que a câmera digital é fixada no corpo da plataforma e, portanto, sujeita a todas as alterações de atitude (direção de voo - kappa; movimentos de asa - ômega; e bico ou calda - phi), conforme destacado por Alves Jr. et al., (2015).

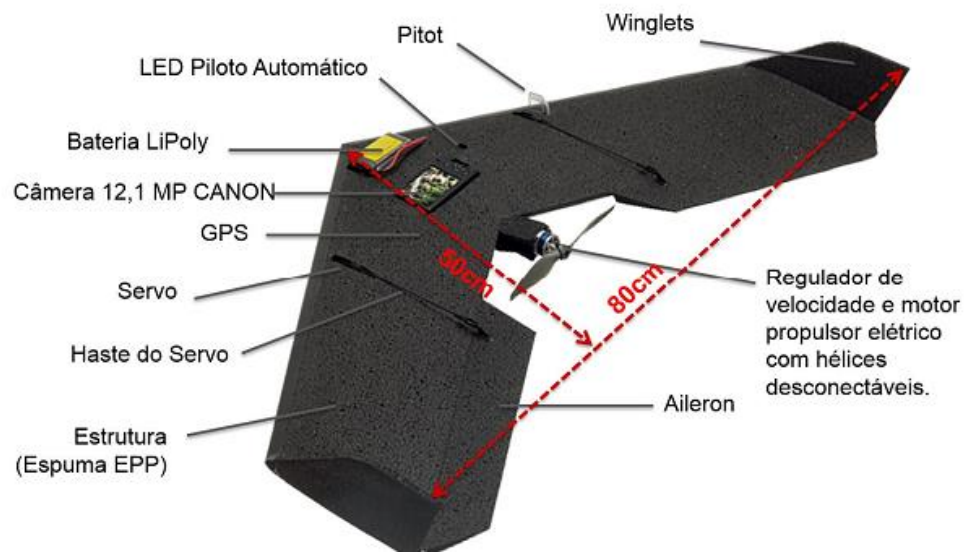

Figura 3 - Vista superior do VANT Swinglet CAM. Adaptado de Sensefly (2012).

O MDS e os mosaicos ortorretificados foram obtidos com uma câmera digital Canon S110, padrão RGB (somente faixas do visível), a bordo do Swinglet CAM. A fim de validar estes produtos, foram realizados trabalhos de campo prévios aos levantamentos aéreos, para escolha e fixação de marcos geodésicos para pós correção da acurácia dos ortomosaicos. Ao todo, foram utilizandos 27 alvos pré-sinalizados, constituídos com uso de sistema GNSS RTK (i.e., GPS geodésico com correção em tempo real), sendo estes distribuídos por toda a área de estudo (Fig. 4). Os pontos foram pintados diretamente no solo exposto nas áreas agrícolas, utilizando-se mistura de cal e água para pintura sobre molde de papelão; este molde 
XVII Simpósio Brasileiro

de Geografia Fisica Aplicada

I Congresso Nacional

de Geografia Física
OS DESAFIOS DA GEOGRAFIA FÍSICA NA FRONTEIRA DO CONHECIMENTO

Instituto de Geociências - Unicamp

Campinas - SP

28 de Junho à 02 de Julho de 2017

continha no centro do alvo um piquete de madeira de $3 \mathrm{~cm}$ (comprimento) x $3 \mathrm{~cm}$ (largura) x $20 \mathrm{~cm}$ (altura), contendo em seu centro um prego para levantamento preciso (1,5 x 16,1 mm).
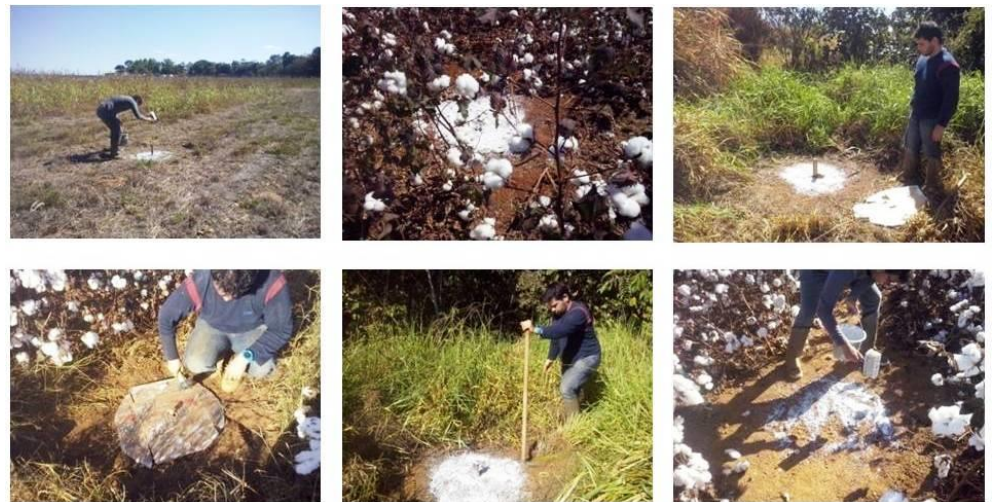

Figura 4. Instalação dos alvos pré-sinalizados, pintados no solo da área de agricultura, para o processamento de georreferenciamento por sistema GNSS.

Com os pontos de apoio marcados em solo (via GNSS ou GPS Geodésico), foi possível a produção de um Modelo Digital de Superfície mais preciso para a área de estudo (com GSD de $10 \mathrm{~cm}$ ). Os blocos aerofotogramétricos foram concebidos no software Emotion, garantindo recobrimento lateral de $40 \%$ e longitudinal de $60 \%$, com o VANT voando a uma altura de $331 \mathrm{~m}$ acima do solo (Fig. 4), totalizando-se um total de 633 fotos georreferenciadas (corrigidas geometricamente e mosaicadas no software Pix4D mapper). Esta metodologia foi baseada em Alves Jr. et al. (2015).

Os resultados foram validados por mais dois trabalhos de campo, nos dias 02 e 04 de janeiro de 2017, com intuito de averiguar in loco os parâmetros hipsométricos, clinográficos e de morfometria das vertentes. Esses ajustes, somados ao uso de software de projetos assistidos por computador - AutoCAD (Autodesk), permitiram a confecção planimétrica de um perfil topográfico em nível de vertente, com escala de exagero vertical de 10 vezes.

\section{Resultados e Discussão}

O desenho experimental interfluvial da área foi sistematizado pela realização dos voos com o Swinglet CAM, obtendo-se um mosaico ortorretificado (projeção ortogonal, com deformações geométricas minimizadas) com resolução espacial de $10 \mathrm{~cm}$; o georreferenciamento foi corrigido com os pontos de apoio em solo, tornando-se uma base geográfica precisa para a construção deste ensaio (Fig. 5). 


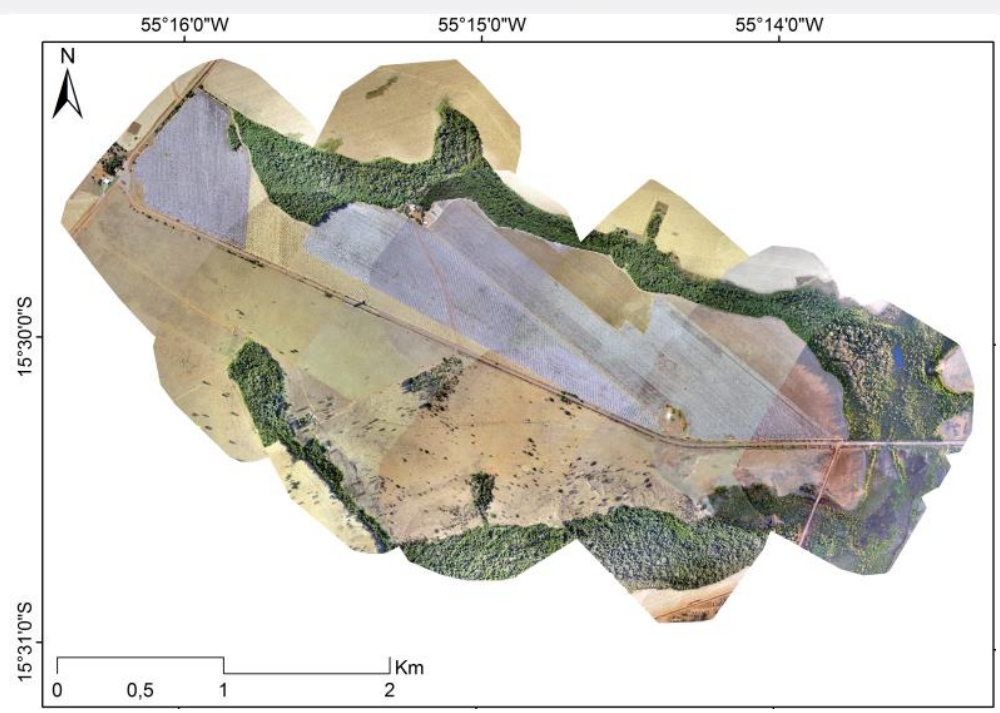

Figura 5. Mosaico aerofotogramétrico obtido com o VANT, representando o desenho experimental da área interflúvial analisada, com resolução espacial de $10 \mathrm{~cm}$, a partir da mosaicagem de 10 planos de voos.

Os processamentos nas imagens coletadas pelo VANT Swinglet CAM possibilitaram a criação do Modelo Digital de Superfície - MDS (método estetoscópico automático, gerado com o software Pix4D mapper), também com resolução espacial de $10 \mathrm{~cm}$, no qual é possível avaliar que a amplitude hipsométrica da área de estudo está contida entre os valores de 576 m e 704 m (Fig. 6). Tal amplitude é corroborada por outros, estudos, que afirmam ser esta a característica da morfoescultura do Planalto e Chapada dos Guimarães (JESUZ, 2014; VASCONCELOS 1998; SANTOS, 2007).

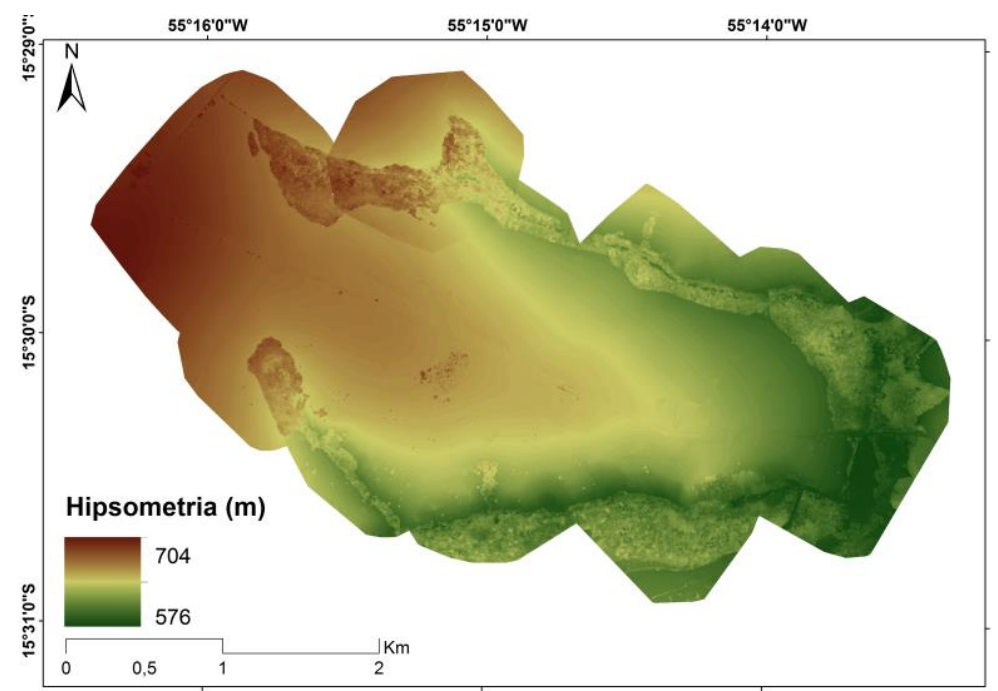

Figura 6: Modelo Digital do Terreno com $10 \mathrm{~cm}$ de resolução espacial. 
Dessa forma, é possível analisar também a formação de um colo de flanco (discutido em GUERRA e GUERRA, 2009), condicionador-base da orientação do fluxo nas vertentes, o qual caracteriza as orientações e desníveis para os cursos d'água nos fundos de vales (Fig. 7). Que derivou uma análise mais profunda sobre sua capacidade de influenciar as determinantes clinográficas, sendo feita em seu centro um perfil de amostragem, que processado por ferramentas cadistas e geoprocessamento, forneceu um perfil de solos com dados do declive das vertentes analisadas.

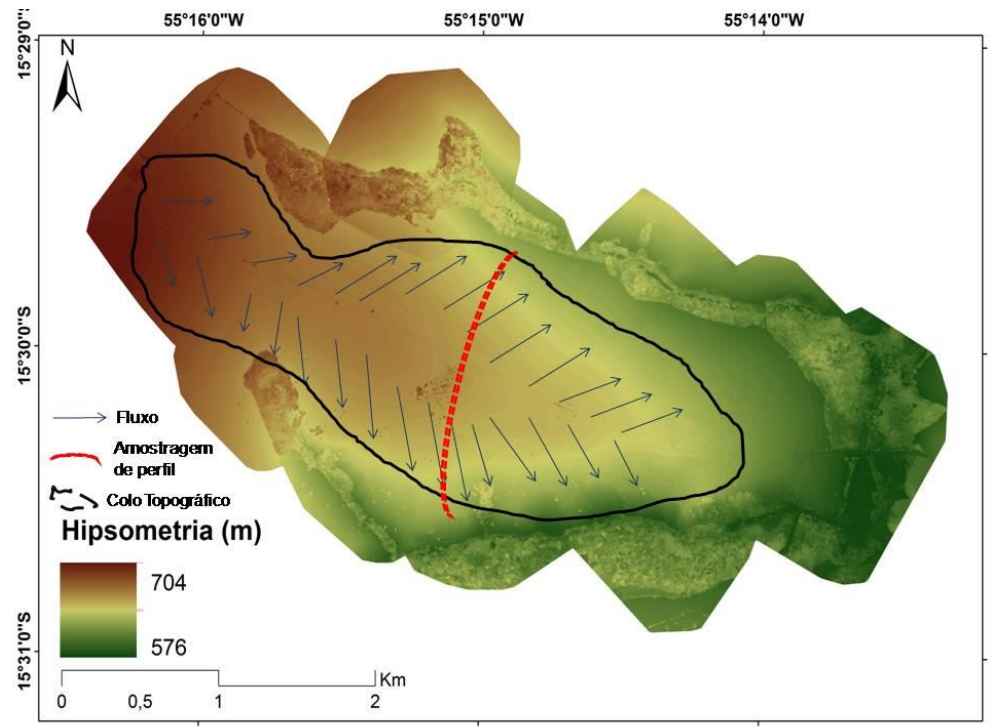

Figura 7 Mapa do colo de flanco, e a tendência de fluxo para os cursos d'água na área de estudo. Com destaque do perfil de solos para exemplificação das determinantes clinográficas.

As interopoções das altitudes geradas pelo MDS foram sistematizadas e planificadas no software AutoCAD, criando condições de representação do interfluvio do desenho experimental, em escala de vertentes, que, ao ser interpretado, forneceu as dimensões clinográficas da área de estudo, visualizadas em um exagero vertical escalar de 10 vezes (Fig. 8).

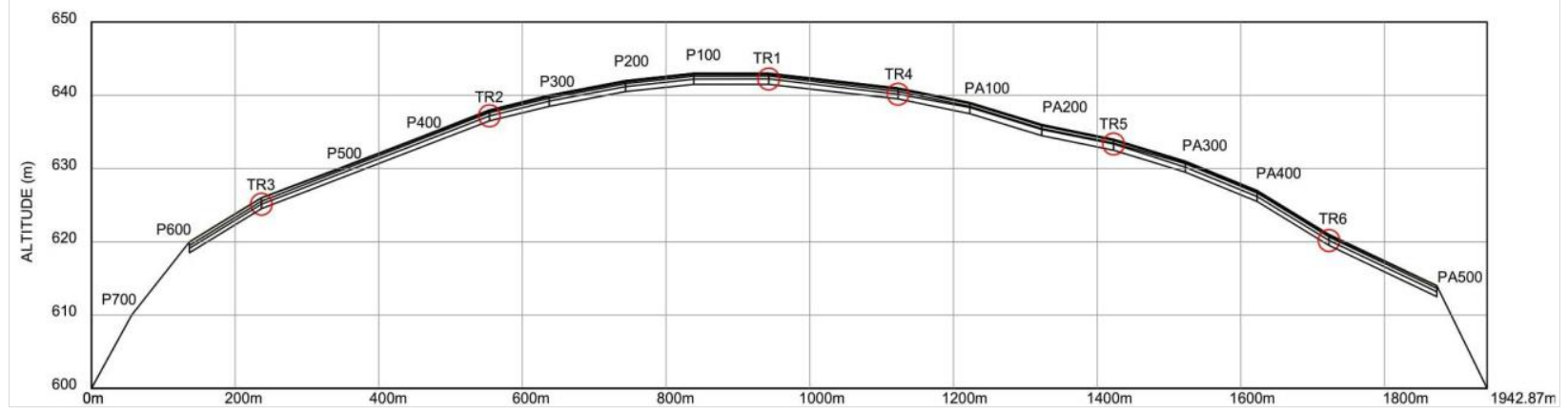

Figura 8: Perfil de representação da morfometria do terreno em condições interfluviais. 
A partir do perfil interfluvial de cerca de 2000 m longitudinal, é possível notar que o terreno analisado é composto por duas calhas de declives diferenciados, sendo a primeira entre a TR1 e a base $600 \mathrm{~m}$,

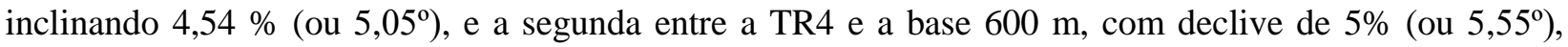
denotando um relevo suave-ondulado, conforme classes da Embrapa (1979) e Pereira (2004).

De acordo com Bertoni e Lombardi Neto (2012), o grau de declive apresenta relação direta com o volume e velocidade das enxurradas que nele acontecem, isto é, "quanto maior o declive, maior a velocidade de escoamento, e, por conseguinte, menor o tempo para a infiltração da água no solo" (BERTONI, 1959, p. 117), o que acelera a velocidade dos processos de formação do runoff (GUERRA, 2010; SALOMÃO, 2010).

Nesse esteio, soma-se também os aspectos da ação social por meio do uso e ocupação da terra, que provocam alterações na estabilidade dos agregados dos solos, e mudanças nas propriedades superficiais e sub-superficiais, especialmente com a supressão da vegetação nativa (bioma Cerrado), e sua conversão em sistemas de plantio e cobertura por forrageiras. O fator preocupante para o recorte espacial analisado, é que $80 \%$ de sua área estão suprimidas pelo agronegócio (agricultura mecanizada e pecuária extensiva) (Fig. 9).

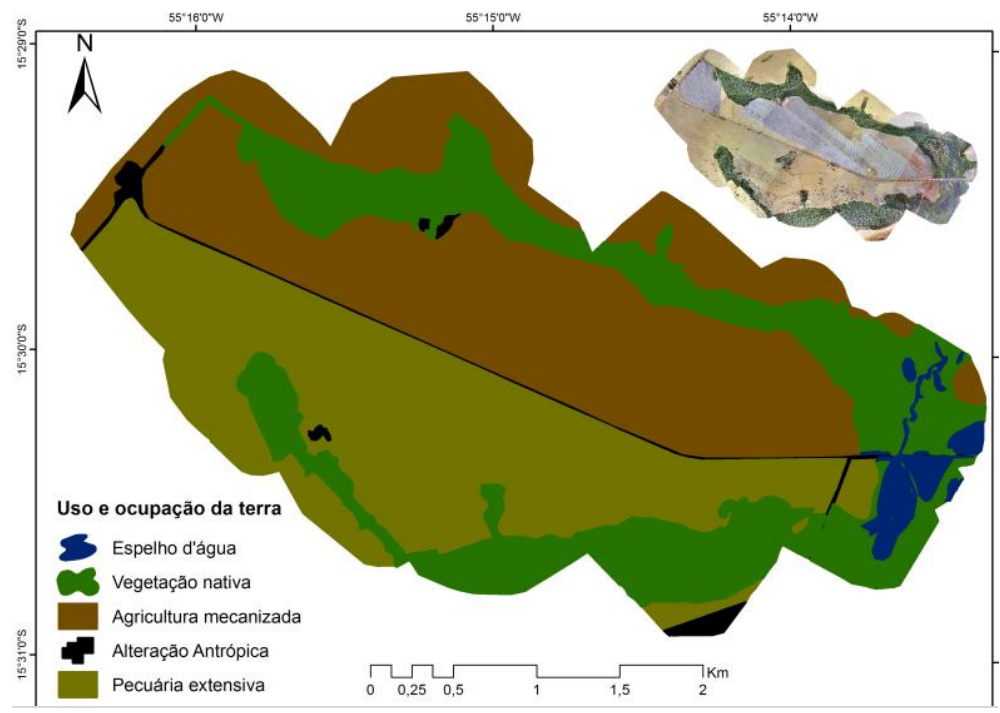

Figura 9: Mapa do uso e ocupação da terra para o recorte analisado, gerado nesta pesquisa a partir do ortomosaico com VANT (voos em agosto de 2016).

Essa supressão foi desencadeada em meados da década de 1970, com a retirada da vegetação nativa, e sua substituição pela pastagem plantada, e sucessões de trocas com as famílias de oleaginosas, malváceas e gramíneas (Tabela ).

Tabela : Descrição do processo de supressão das nascentes analisadas. 


\begin{tabular}{|c|c|c|}
\hline \multirow{3}{*}{ Uso } & \multirow{2}{*}{$\begin{array}{l}\text { XVII Simpósio Brasileiro } \\
\text { de Geografia Fisica Aplicada } \\
\text { I Congresso Nacional } \\
\text { de Geografia Física }\end{array}$} & \multirow{2}{*}{$\begin{array}{l}\text { OS DESAFIOS DA GEOGRAFIA FÍSICA NA FRONTEIRA DO CONHECIMENTO } \\
\text { Instituto de Geociências - Unicamp } \\
\text { Campinas - SP } \\
28 \text { de Junho à } 02 \text { de Julho de } 2017\end{array}$} \\
\hline & & \\
\hline & Período & Culturas e Manejo Geral \\
\hline \multirow[b]{2}{*}{ Pecuária } & $1970 / 1975$ & Retirada da vegetação nativa. \\
\hline & $1975 / 2016$ & Pastagem de brizantã, $1,5 \mathrm{t} \mathrm{ha}^{-1}$ de calcário dolomítico a cada 3 anos. \\
\hline \multirow{6}{*}{$\begin{array}{l}\text { Área de } \\
\text { Agricultura }\end{array}$} & $1970 / 1975$ & Retirada da vegetação nativa. \\
\hline & $1975 / 1990$ & Pastagem de brizantã, $1,5 \mathrm{t} \mathrm{ha}^{-1}$ de calcário dolomítico a cada 3 anos. \\
\hline & $1990 / 1992$ & Retirada da pastagem, e plantio de soja convencional. \\
\hline & $1992 / 2004$ & Replantio de pastagem de brizantã, $1,5 \mathrm{t} \mathrm{ha}^{-1}$ de calcário dolomítico a cada 3 anos. \\
\hline & $2004 / 2009$ & $\begin{array}{l}\text { Retirada da pastagem, e plantio de soja, com rotatividade do milho safrinha em sistema } \\
\text { convencional. }\end{array}$ \\
\hline & $2009 / 2016$ & Implementação do algodão safrinha no sistema de soja/milho. \\
\hline
\end{tabular}

Em estudos realizados por Jesuz (2014), o autor debate sobre os processos de erosões lineares e mecânicos derivados das ações de supressão vegetal do Cerrado, pelo uso e ocupação da terra com o agronegócio, ressaltando que áreas com agricultura mecanizada, em sistema de rotação safra (Out-Jan) e safrinha (FevJun), apresentam grande propensão aos processos de erosão laminar; isto se deve ao fato da cobertura do solo está relacionada diretamente ao estágio vegetativo das plantas, especialmente a cobertura foliar, sendo que a troca de culturas acontece no período de maiores eventos pluviométricos com chuvas torrenciais. Essa situação de supressão da vegetação nativa, associada aos parâmetros naturais da paisagem, faz com que a área estudada seja semelhante a outras paisagens encontradas em Mato Grosso, com predominância do agronegócio.

\section{Considerações Finais}

A utilização de novas tecnologias, como um VANT equipado com sensores imageadores, para a obtenção de dados com alta resolução espacial, associados aos métodos tradicionais de análise da paisagem, se mostrou bastante favorável para avaliar os impactos ambientais em sistemas produtivos inadequados, bem como respectiva orientação de manejo e panejamento. Assim, os resultados obtidos nesse ensaio são capazes de subsidiar de forma concreta uma boa parametrização da paisagem nos estudos que envolvam as constantes físicas e antrópicas. E, associando-as com imagens aéreas e produtos correlatos (MDS e mapa de uso do solo, por exemplo), condicionam uma boa alternativa temporal e financeira para trabalhos que envolvam dinâmicas acentuadas na paisagem.

\section{Bibliografia}

ALMEIDA, J. Normas de Segurança para Implantação dos VANT Civis no Espaço Aéreo Brasileiro: uma nova abordagem. Conexão SIPAER, v. 2, n. 1, 2010.

ALVES JÚNIOR, L. R. Análise de produtos cartográficos obtidos com câmera digital não métrica acoplada a um Veículo Aéreo Não Tripulado em áreas urbanas e rurais no estado de Goiás. 2015. Dissertação. Programa de Pós-Graduação em Geografia, do Instituto de Estudos Socioambientais da Universidade Federal de Goiás, 2015. 
BERTONI, J. O espaçamento dos terraços em culturas anuais, determinado em função das perdas por erosão. Bragantia. IAC, Campinas. V. 18(1) . p.113-140, 1959.

BERTONI, J.; LOMBARDI NETO, F. Conservação do Solo. - 8a . ed. - São Paulo: Ícone, 2012.

BOEGH E; THORSEN M; BUTTS M. B; HANSEN S; CHRISTIANSEN J. S; ABRAHAMSEN P; HASAGER C. B; JENSEN N. O; VAN DER KEUR P; REFSGAARD J. C; SCHELDE K; SOEGAARD H; THOMSEN A. Incorporating remote sensing data in physically based distributed agro-hydrological modelling. Journal of Hydrology 287:279-299, 2004.

COLOMINA, I.; MOLINA, P. Unmanned aerial systems for photogrammetry and remote sensing: A review. ISPRS Journal of Photogrammetry and Remote Sensing, 92, 79-97, 2014.

DANDOIS, J. P.; OLANO, M.; ELLIS, E. C. Optimal Altitude, Overlap, and Weather Conditions for Computer Vision UAV Estimates of Forest Structure. Remote Sensing, 7, 13895-13920, 2015.

DIAS, H. G.; ROSSA, A. A. Considerações Sobre o Emprego de Veículos Aéreos não Tripulados (Vant) Armados à Luz do Direito Internacional dos Conflitos Armados. Coleção Meira Mattos, Rio de Janeiro, v. 9, n. 34, p. 189-200, jan./abr. 2015.

EMPRESA BRASILEIRA DE PESQUISA AGROPECUÁRIA - EMBRAPA. Súmula da 10a Reunião Técnica de Levantamento de Solos. Serviço Nacional de Levantamento e Conservação de Solos. Rio de Janeiro, 1979.

FLENER, C.; VAAJA, M.; JAAKKOLA, A.; KROOKS, A.; KAARTINEN, H.; KUKKO, A.; KASVI, E.; HYYPPÄ, H.; HYYPPÄ, J.; ALHO, P. Seamless Mapping of River Channels at High Resolution Using Mobile LiDAR and UAV-Photography. Remote Sensing, 5, 6382-6407, 2013.

GUERRA, A. J. T. O início do processo erosivo. In: GUERRA, A. T. J.; SILVA, A. S.; MACHADO, R. G. (Orgs.). Erosão e conservação dos solos: conceitos, temas e aplicações. $6^{\mathrm{a}}$ ed. - Rio de Janeiro: Bertrand Brasil, 2010, p. $17-50$.

GUERRA, A. T. GUERRA, A. J. T. Novo Dicionário Geológico-Geomorfológico - $7^{\mathrm{a}}$ ed. - Rio de Janeiro: Bertrand Brasil, 2009.

JESUZ, C. R.; ITO, J. B. B,; PETER, Z. Erosões mecânicas na bacia hidrográfica do rio Tenente Amaral, Jaciara - MT, e suas determinantes socioambientais. Revista Mato-Grossense de Geografia - Cuiabá - n. 16 - p. $89-105$ - jan/jun 2013.

JESUZ, C. R. de. Estudo geomorfológico e a análise dos processos de erosão mecânica na bacia hidrográfica do rio Tenente Amaral - MT. 2014, fls. 142. Dissertação. Universidade Federal de Mato, Instituto de Ciências Humanas e Sociais, Programa de Pós-Graduação em Geografia, Cuiabá, 2014. 
LATRUBESSE, E. M., RODRIGUES, S. C., MAMEDE, L. Sistema de classificação e mapeamento geomorfologicos: Uma nova proposta. Geosul. vol. 14 (17), 682-687. 1998.

LUCIEER, A.; TURNER, D.; KING, D. H.; ROBINSON, S, A. Using an Unmanned Aerial Vehicle (UAV) to capturemicro-topography of Antarctic moss beds. International Journal of Applied Earth Observation and Geoinformation, 2013.

MONTEIRO, C. A. Geossistema: a história de uma procura. São Paulo: Contexto, 2001.

NÄSI, R.; HONKAVAARA, E.; LYYTIKÄINEN-SAARENMAA, P.; BLOMQVIST, M.; LITKEY, P.; HAKALA, T.; VILJANEN, N.; KANTOLA, T.; TANHUANPÄÄ, T.; HOLOPAINEN, M. Using UAV-Based Photogrammetry and Hyperspectral Imaging for Mapping Bark Beetle Damage at Tree-Level. Remote Sensing, 7, 15467-15493, 2015.

NIETHAMMER, U.; JAMES, M. R.; ROTHMUND, S.; TRAVELLETTI, J.; JOSWIG, M. UAV-based remote sensing of the Super-Sauze landslide: Evaluation and results. Engineering Geology, 128, 2-11, 2012.

NIKOLAKOPOULOS, K. G.; KAVOURAA, K.; DEPOUNTISA, N.; ARGYROPOULOSA, N.; KOUKOUVELASA, I.; SABATAKAKISA, N. Active landslide monitoring using remote sensing data, GPS measurements and cameras on board UAV. In: Third International Conference on Remote Sensing and Geoinformation of the Environment (RSCy2015), 2015.

OUÉDRAOGO, M. M.; DEGRÉ, A.; DEBOUCHE, C.; LISEIN, J. The evaluation of unmanned aerial systembased photogrammetry and terrestrial laser scanning to generate DEMs of agricultural watersheds. Geomorphology 214, 339-355, 2014.

PEREIRA, L. C. Avaliação da aptidão agrícola das terras: proposta metodológica. --Jaguariúna: Embrapa Meio Ambiente, 2004.

REATTO, A.; MARTINS, E. S. FARIAS, M. F. R.; DA SILVA, A. V.; DE CARVALHO JR., O. A. Mapa Pedológico digital SIG atualizado do Distrito Federal, escala 1:100.000 e uma Síntese do Texto Explicativo. Planaltina: Embrapa Cerrados, 2004. 31 p.

SALOMÃO, F. X. T. Controle e Prevenção dos Processos Erosivos. In: GUERRA, A. J. T; SILVA, A. S; BOTELHO, R. G. M. (Org.). Erosão e conservação dos solos: conceitos, temas e aplicações. $6^{\text {a }}$ ed. - Rio de Janeiro: Bertrand Brasil, 2010. p. 229 - 265.

SANO, E. E.; ASSAD, E. D.; ORIOLI, A. L. Monitoramento da ocupação agrícola. In: ASSAD, E. D.; SANO, E. E. Sistema de informações geográficas: aplicações na agricultura. Planaltina: EMBRAPA - CPAC. p.157-70, 1993.

SANTOS, V. S. Análise ambiental integrada do componente solo como subsídio para avaliação da sustentabilidade da Bacia Hidrográfica do Rio Tenente Amaral em Jaciara - Mato Grosso - Brasil. 2007, fls. 
191. Tese. Universidade Federal de São Carlos, Centro de Ciências Biológicas e da Saúde, Programa de PósGraduação em Ecologia e Recursos Naturais. São Carlos, 2007.

SCHMUGGE T.J., KUSTAS W.P., RITCHIE J.C., JACKSON T.J., RANGO, A. (2002) Remote sensing in hydrology. Adv Water Resour 25,1367-1385.

VASCONCELOS, T. N. N. Interpretação morfopedológica da bacia do rio Tenente Amaral - Jaciara-MT: condição básica para sua caracterização ambiental. 1998, fls.160. Dissertação. Universidade Federal de Mato Grosso, Instituto de Ciências Biológicas, Programa de Pós-Graduação em Ciências Biológicas. Cuiabá, 1998.

WWF BRASIL. Monitoramento das alterações da cobertura vegetal e uso do Solo na Bacia do Alto Paraguai Porção Brasileira - Período de Análise: 2002 a 2008. Iniciativa: CI - Conservação Internacional, ECOA Ecologia e Ação, Fundación AVINA, Instituto SOS Pantanal, WWF- Brasil. Brasília, 2009.

WWF-BRASIL. Monitoramento das alterações da cobertura vegetal e uso do Solo na Bacia do Alto Paraguai - Porção Brasileira - Período de Análise: 2008 a 2010. Iniciativa: CI - Conservação Internacional, ECOA Ecologia e Ação, Fundación AVINA, Instituto SOS Pantanal, WWF- Brasil. Brasília, 2011.

\section{Agradecimentos:}

À CAPES, pelo apoio na aquisição do VANT junto ao Programa de Pós-Graduação em Geografia da Universidade Federal de Goiás (Pró-equipamentos 2012 e 2014). Ao WWF (Programa Ecodrones), pelo apoio na manutenção do VANT. À Santiago \& Cintra, Sensefly e Pix4D, pela parceria com o LAPIG /UFG. O terceiro autor é bolsista de produtividade pelo CNPq. 